\title{
Improved Simulation of Regional Climate by Global Models with Higher Resolution: Skill Scores Correlated with Grid Length*
}

\author{
I. G. WATTERSON \\ CSIRO, Aspendale, Victoria, Australia
}

(Manuscript received 16 October 2014, in final form 14 April 2015)

\begin{abstract}
The current generation of climate models, as represented by phase 5 of the Coupled Model Intercomparison Project (CMIP5), has previously been assessed as having more skill in simulating the observed climate than the previous ensemble from phase 3 of CMIP (CMIP3). Furthermore, the skill of models in reproducing seasonal means of precipitation, temperature, and pressure from two observational datasets, quantified by the nondimensional Arcsin-Mielke skill score, appeared to be influenced by model resolution. The analysis is extended to 42 CMIP5 and 24 CMIP3 models. For the combined skill scores for six continents, averaged over the three variables and four seasons, the correlation with model grid length in the 66-model ensemble is -0.73 . Focusing on the comparison with ERA-Interim data at higher resolution and with greater regional detail, correlations are nearly as strong for scores over the ocean domain as for land. For the global domain (excluding the Antarctic cap), the correlation of the overall skill score with grid length is -0.61 , and it is nearly as strong for each variable. For most tests the improved averaged score of CMIP5 models relative to those from CMIP3 is largely consistent with their increased resolution. However, the improvement for precipitation and the correlations with length are both smaller if rmse is used as a metric. They are smaller again using the GPCP observational data, as the regional detail from a high-resolution model can lead to larger differences when compared to relatively smooth observational fields.
\end{abstract}

\section{Introduction}

The Fifth Assessment Report of the Intergovernmental Panel on Climate Change and recent national assessments of future climate including those for the United States (Melillo et al. 2014) and Australia (CSIRO and Bureau of Meteorology 2015) have been largely based on climate simulations submitted to phase 5 of the Coupled Model Intercomparison Project (CMIP5). There are various studies documenting the quality of the simulations of the recent past climate. Flato et al. (2013) compared the CMIP5 simulations with observational data on the global scale and concluded that overall the latest generation of climate models had improved over

\footnotetext{
* Supplemental information related to this paper is available at the Journals Online website: http://dx.doi.org/10.1175/JCLI-D-1400702.s1.

Corresponding author address: I. G. Watterson, CSIRO, Marine and Atmospheric Research, Station St., Aspendale, Victoria 3195, Australia.

E-mail: ian.watterson@csiro.au
}

models from the earlier phase 3 of CMIP (CMIP3). They also noted that at regional scales precipitation (or pr, in the CMIP5 nomenclature) is not simulated as well as (surface air) temperature (tas), although the assessment of pr, in particular, is hampered by observational uncertainties. Regional studies, such as Sheffield et al. (2013) for North America, Yin et al. (2013) for South America, and CSIRO and Bureau of Meteorology (2015) for Australia, find a considerable range of skill in matching observed climatological features across the CMIP5 ensemble of models.

Flato et al. (2013) attributed the improved skill in some newer models both to improved representation of physical processes and to increased resolution. This is consistent with numerous previous studies, including those of Boyle and Klein (2010), Delworth et al. (2012), and Neale et al. (2013), who documented overall improvements when global models are run at higher resolution. Recently, Watterson et al. (2014) assessed the relationship between the horizontal resolution of models and their nondimensional "Arcsin-Mielke" skill score for simulated fields over both individual continents and global land across a combined CMIP3 
and CMIP5 ensemble. The correlation coefficient $(r)$ between resolution (quantified by average grid length) and the overall score for precipitation was -0.61 . (Note that higher scores represent an improvement, for this metric, and relate to decreased grid length, given the negative $r$ values. In comparing $r$ values, it will be implicit that the magnitude is intended.) Correlations for tas $(r=-0.59)$ and mean sea level pressure ( $\mathrm{psl} ; r=-0.61)$ were similar, while that for the three-variable average was higher, $r=-0.71$. Furthermore, the improvement in average scores from CMIP3 to CMIP5 was quite consistent with the increase in resolution, given the regression lines fitted to the data. A difference between average scores for CMIP5 Earth system (ES) models, which include some biogeochemical processes, and the other atmosphere-ocean (AO) models, was also consistent with the lower resolution of the ES models, on average. (Note that the additional ES processes would not be expected to have a major impact on the simulated climate, since they mostly relate to variables such as land cover and carbon dioxide concentrations that are prescribed in these simulations.) How much the improvements in scores reflect those in modeling systems in addition to direct effects of model resolution is unclear, however.

The CMIP5 ensemble used by Watterson et al. (2014) consisted of 25 models (with available data) that were used for simulations extending through the twenty-first century, hence excluding the highest-resolution model MIROC4h (Sakamoto et al. 2012). Subsequently data became available for a further 16 such models, including three with resolution approaching that of MIROC4h. Recently, ERA-Interim data used in some of the tests also became available at higher resolution. This allows the ability of the new climate models in simulating regional details in that data to be better tested. Restricting the domains to the continents can potentially result in relatively high skill scores for models that better resolve coastlines and land-sea contrasts in climatological fields. Could the above correlations have been enhanced by this? Of course, skills over the ocean, and combined land-ocean domains are also of considerable interest.

Given the assessment of Flato et al. (2013), the similarity of the above correlation for pr to those for tas and psl is somewhat surprising. Pope and Stratton (2002), Mass et al. (2002), Mishra et al. (2014), and others have found that improvement in the simulation of precipitation (on various time scales) with model resolution can be modest. Gleckler et al. (2008) noted the potential dependence of the relative ranking of models for precipitation on the observational dataset, the metric used, and the resolution of the analysis grid. A high-resolution model is more susceptible to the "double-penalty problem," as it has been referred to in weather forecasting (e.g., Wernli et al. 2009), when a metric penalizes a simulation for missing a feature of high precipitation in the observational data and also for simulating it at a location where the observational data have less precipitation. For the climatological means, Watterson et al. (2014) noted somewhat smaller correlations for alternative skill metrics and precipitation datasets, and this warrants further assessment, particularly given that the reanalysis pr is not directly based on observations.

The initial aims of this paper are to update the results of Watterson et al. (2014) with the additional CMIP5 models, to make use of the higher-resolution ERAInterim data, and to broaden the tests to include the ocean domain. These aims are addressed in the section 4 , following a section detailing the data and metrics, and a section presenting features simulated by the additional models and also variations between all the models. Section 5 considers further the implications of the regression lines relating skill to model grid length, and the dependence of model rankings on the metric and observational data. Section 6 focuses on the assessment of precipitation over land, including a regional illustration, and is followed by the conclusions.

\section{Datasets and methods}

\section{a. Models}

For this assessment of present climate skill, 17 newer models are added to the CMIP5 ensemble used by Watterson et al. (2014), including MIROC4h, with a representative grid length of $50 \mathrm{~km}$. This length was determined by dividing Earth's surface area by the number of points on the model's global surface data grid, then taking the square root. The new model CMCC-CM (Scoccimarro et al. 2011) has a grid length of $67 \mathrm{~km}$, smaller than the $96 \mathrm{~km}$ of the best-resolved model of the 25-member ensemble, NCAR's CCSM4. The new model CESM1(CAM5) matches that resolution; see Hurrell et al. (2013) for a description of the NCAR models. The new model EC-EARTH (Hazeleger et al. 2012), based on the ECMWF's seasonal prediction model, has a slightly larger length of $100 \mathrm{~km}$. Each of these is categorized as an AO model (following Flato et al. 2013). The remaining 13 additional climate models all have atmospheric components that are in common or closely based on those of the other models \{e.g., the NCAR Community Earth System Model, version 1, with biogeochemistry [CESM1(BGC)]\}, and with grid lengths ranging from 96 to $333 \mathrm{~km}$. (For brevity, the full list of the 42 CMIP5 models considered here and the list of the 24 CMIP3 models are given in Tables S1 and S2 of the 
TABLE 1. Observational datasets used in the skill assessments, for both the combined variable results and additional analysis. The name by which the set is referred to, the variables, and the full name of the source are given (CRU TS1.0 is version 1.0 of the CRU time series data). These are followed by the spacing (in both latitude and longitude) of the grid, the representative grid length (km), and the published reference; see text for further details. Another field used is the smoothed ERA-H, denoted ERA-S (see section 5c).

\begin{tabular}{|c|c|c|c|c|c|c|}
\hline Name & Variables & Source & Grid & Grid length & Years & Reference \\
\hline \multicolumn{7}{|c|}{ Combined datasets } \\
\hline ERA-H & tas, pr, psl & ERA-Interim & $0.75^{\circ}$ & 66 & 1979-2008 & Dee et al. (2011) \\
\hline ERA & tas, pr, psl & ERA-Interim & $1.5^{\circ}$ & 133 & 1979-2008 & Dee et al. (2011) \\
\hline \multirow[t]{2}{*}{$\mathrm{CRU}$} & tas, pr & CRU TS1.0 & $2^{\circ}$ & 177 & $1961-90$ & New et al. (1999) \\
\hline & psl & ERA-40 & $2.5^{\circ}$ & 220 & $1957-2002$ & Uppala et al. (2005) \\
\hline \multicolumn{7}{|l|}{ Other data } \\
\hline CRU-H & $\mathrm{pr}$ & CRU TS1.0 & $0.5^{\circ}$ & 44 & $1961-90$ & New et al. (1999) \\
\hline GPCP & pr & GPCPv2.2 & $2.5^{\circ}$ & 220 & 1979-2008 & Adler et al. (2003) \\
\hline GPCP-H & pr & GPCP 1dd & $1^{\circ}$ & 89 & 1996-2014 & Huffman et al. (2001) \\
\hline
\end{tabular}

supplementary material; expansions of model name acronyms are available online at http://www.ametsoc.org/ PubsAcronymList.) The 23 CMIP5 models categorized as ES are indicated there. For each CMIP5 model, the primary simulation of the "historical" experiment is used. Even multidecadal averages of simulations can be somewhat affected by unforced variability, and Watterson et al. (2014) assessed this using a 10-member ensemble of the CSIRO Mk3.6 model (Rotstayn et al. 2012). The ensemble is again used, along with two additional simulations from both EC-EARTH and CESM1(CAM5). Further details of the models and experiments, for both CMIP3 and CMIP5, can be found in the supplementary material and Flato et al. (2013).

\section{b. Observational data}

Watterson et al. (2014) used two sets of observational data for the three variables tested. As in an earlier CMIP3 analysis, one set included tas and pr from the CRU land dataset for 1961-90 (New et al. 1999) aggregated to a $2^{\circ}$ grid, which includes data for numerous islands (see Fig. S1 in the supplementary material). The other was the reanalysis dataset ERA-Interim (Dee et al. 2011) for 1979-2009, used for all three variables. For convenience, these sets are denoted CRU and ERA, with further details, including representative grid lengths, given in Table 1.

The ERA data are on a $1.5^{\circ}$ data grid, interpolated from the raw model grid (of around $0.7^{\circ}$; P. Berrisford 2014, personal communication). Data on a finer grid of $0.75^{\circ}$ are now available and seasonal climatologies of the three fields have been formed for the same period, and denoted ERA-H (for high resolution). Consistent with an interpolation, the data on the lower-resolution grid are basically a subset of those from the finer grid; however, there are some differences in tas and pr values at coastal points, following from a consideration of the surface type (land or ocean), for those variables. The representative length for the new grid, $66 \mathrm{~km}$, is similar to that of the best-resolved models, and hence will allow regional detail to be better tested than before. This detail is at least partly dependent on the reanalysis model, but the tas and psl fields are used in numerous studies and by Flato et al. (2013). The precipitation field is generated by the model but is driven by a state-of-theart representation of the weather and surface conditions. Simmons et al. (2010) showed that the ERA pr compares well with standard alternative datasets over the continents. Indeed, Yatagai et al. (2012) note considerable variations among datasets that are based on gauge observations, particularly in regions with large topographic variation. Over the ocean all gridded pr data are largely inferred [see Gruber and Levizzani (2008) for a comprehensive assessment]. Since the comparison between results for land and ocean domains is a focus of section 4, the main dataset is ERA-H, which has the advantage of a consistent resolution and analysis process across the globe. However, the conclusions regarding skill are with respect to similarity to the reanalysis data, rather than true observations.

Additional pr data considered, particularly in section 6, are CRU on the full $0.5^{\circ}$ grid, denoted CRU-H, and two versions of GPCP (Adler et al. 2003). The version 2.1 on a $2.5^{\circ}$ grid considered by Watterson et al. (2014) is updated to version 2.2, again using a climatology from years 1979 to 2008. The second version of Huffman et al. (2001) is on a $1^{\circ}$ grid (1dd) and is hence denoted here as GPCP-H. An 18-yr seasonal climatology was constructed from time-averaged fields from October 1996 through September 2014, provided by Angeline Pendergrass (2014, personal communication).

\section{c. Skill metrics}

The skill test is limited to a comparison of timeaveraged, or climatological, gridded fields of tas, pr, and psl for the standard three-month seasons. The 
CMIP5 historical simulations extend to the nominal year 2005, and averages over the 30-yr period 1975-2004 have been constructed, with the December-February season extending to February 2005. In these simulations there is no relationship between the weather and unforced variability, even on interdecadal time scales, to the actual variability, so the choice of period for the datasets is not crucial.

Following Watterson et al. (2014), the main metric is the nondimensional Arcsin-Mielke measure $M$. In essence it is the mean-square error (mse), nondimensionalized by the spatial variance of the field. Specifically, for the mse between the model field $X$ and observed field $Y$,

$M=\left(\frac{2}{\pi}\right) \arcsin \left[1-\frac{\text { mse }}{V_{X}+V_{Y}+\left(G_{X}-G_{Y}\right)^{2}}\right] \times 1000$,

where $V$ is the variance and $G$ the mean, with all statistics calculated over the domain under consideration. Area weighting of the points on the observational grid are used, with each model field linearly interpolated to that grid. The final factor provides a skill score that has a maximum possible value (for mse $=0$ ) of 1000 "points," while a zero score indicates no skill (negative values are rare in this context). The arcsin transformation, applied to a measure of Mielke (1991), means that the deviation from unity asymptotes to the square root of mse (or rmse). As discussed by Watterson (1996), in the case of fields with the same variance and mean, the Mielke measure is the same as the spatial correlation coefficient ( $r$ ), as used by Flato et al. (2013, their Fig. 9.6). Both $M$ and $r$ include a scaling of errors by spatial variations. In addition to being unity only for identical fields, $M$ has the advantage of allowing greater differentiation for tas, or other fields with a large variation relative to rmse. That statistic, or root-mean-square difference, will be used as a second measure of agreement between the model and observed fields. The mean absolute error (mae) will also be briefly considered.

Given multiple simulations of a model, the average of the resulting skill scores would provide a more accurate measure of skill than any individual score with regard to unforced variability. The score for each primary simulation can be considered to have a statistical uncertainty relative to such an average score. With a 95th percentile range of a normal distribution being approximately two standard deviations (SDs) either side of the mean, that half-range is an indication of the statistical uncertainty of a single value. The SD is estimated here using the 10 values calculated from the CSIRO Mk3.6 ensemble, which is supported by the resulting ranges being comparable to those of the smaller EC-EARTH and CESM1(CAM5) ensembles considered. The estimated uncertainty of a value is twice this $\mathrm{SD}$. It is generally small compared to the differences between models.

Watterson et al. (2014) considered six idealized continental domains (omitting Antarctica), and also a (near) global land (GL) domain, including land points represented by the observational data north of $60^{\circ} \mathrm{S}$. (The domains used are shown in Fig. S1.) For the new tests in this study, the same ERA-Interim GL domain is used, aside from the higher resolution. The same southern boundary is used for the global ocean (GO) domain. Tests are also made for the combined GL and GO domain, or GLO. The choice for land was consistent with the aim of testing variables and domains important to climate impacts. Excluding the southern cap also avoids a region of considerable observational uncertainty.

\section{Climatological fields}

\section{a. Regional detail in the datasets}

The new datasets allow analysis of climatological detail, at higher resolution, over the globe. Cases of high similarity between selected models and ERA-H (ERAInterim, on the $0.75^{\circ}$ grid) are illustrated here. Starting with precipitation, pr 30-yr means for the SeptemberNovember (SON) season are plotted for the (near) globe in Fig. 1. Both ERA-H (Fig. 1a) and CESM1(CAM5) (Fig. 1c) exhibit large contrasts in the low latitudes, with rain rates exceeding $30 \mathrm{~mm} \mathrm{day}^{-1}$ in the far northeast of South America, and rates below $0.1 \mathrm{~mm} \mathrm{day}^{-1}$ over the Sahara and over the ocean just to the west of subtropical deserts. Contrasts over the midlatitude land and ocean are also similar in both fields. High values occur along the Himalayas and along the convergence zones of the Pacific and Atlantic Oceans, although with some differences in position in the western Pacific. In fact, this case illustrates the highest level of agreement using $M$ with ERA-H pr for any season over the GLO domain among all CMIP5 models, with an $M$ value of 727 points. The rmse is $1.12 \mathrm{~mm} \mathrm{day}^{-1}$, which is also the best result for this case. Scores for the other two simulations from CESM1(CAM5) are rather similar, 717 and 718 points, and 1.15 and $1.13 \mathrm{~mm} \mathrm{day}^{-1}$.

Interestingly, the use of the finer-resolution ERA-H dataset, rather than the earlier $1.5^{\circ}$ (ERA) version, does not result in notably different scores. In the Fig. 1 case the difference is 1 point or $0.003 \mathrm{~mm} \mathrm{day}^{-1}$. This is because the biases at the common points (other than on some coastal points noted above) remain the same, so skill scores differ only because intervening points are included in the $0.75^{\circ}$ case. The mse for a domain, as well as the other terms in the formulation of $M$, do not differ 

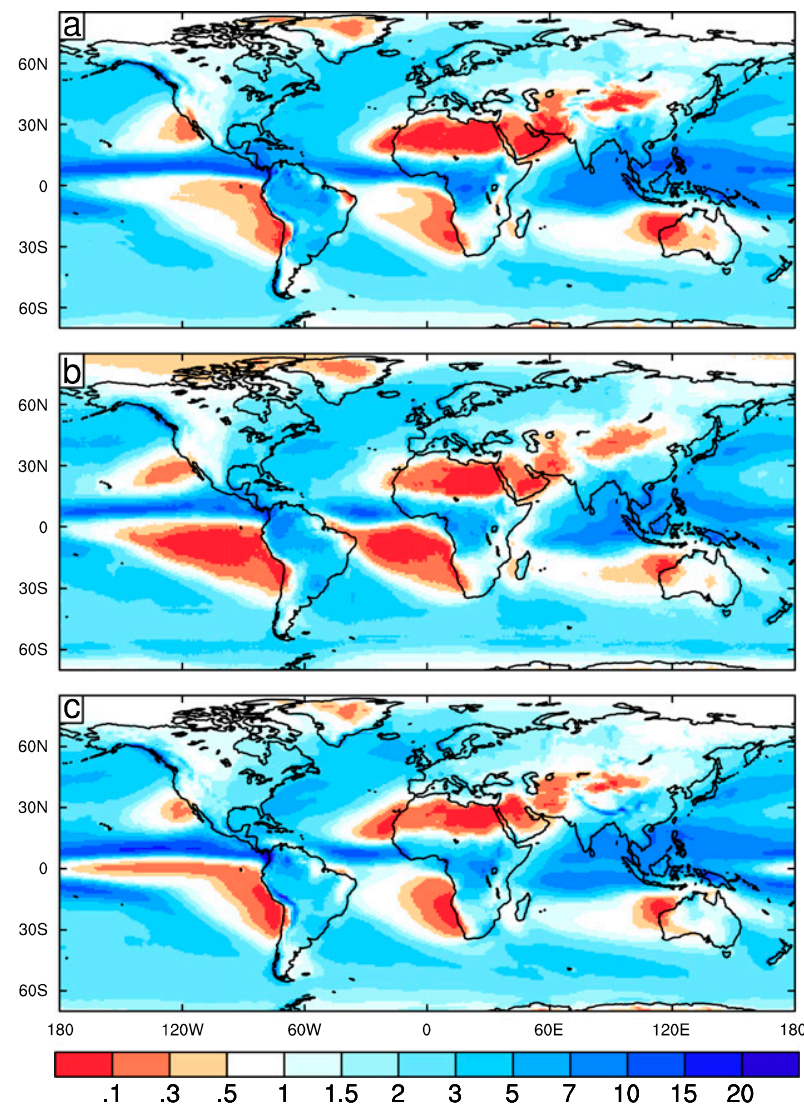

FIG. 1. Climatological mean pr for September-November $\left(\mathrm{mm} \mathrm{day}^{-1}\right.$ ): (a) ERA-Interim, (b) GPCP-H, and (c) simulation from CESM1(CAM5).

significantly. However, scores and rankings can certainly depend on the observational dataset. Figure $1 \mathrm{~b}$ shows the GPCP-H observational field. CESM1(CAM5) has a lower $M$ score for this, 642 points. There are differences between ERA-H and GPCP-H, with an $M$ score for agreement of 714. Further consideration of the dependence on dataset is deferred to sections 5 and 6 .

The highest $M$ score for tas from ERA-H over GLO is 931, achieved by MPI-ESM-LR [a model assessed by Watterson et al. (2014)], in December-February (DJF). The new version MPI-ESM-MR (Giorgetta et al. 2013) has more atmospheric vertical levels and has higher scores in the other seasons. Both these models have the grid length $166 \mathrm{~km}$ and so cannot fully resolve some of the detail of the ERA-Interim field. Nevertheless they simulate both the lower temperatures over orography and the large-scale spatial variation very well. See Fig. S2 in the supplementary material for maps for DJF.

The top score for psl over GLO is 882 and, as for tas, this is for CESM1(CAM5) in SON. In the other seasons
EC-EARTH is best, and the DJF case is illustrated in Fig. S3 of the supplementary material. Much of the smallerscale detail in the ERA-H psl field is over high orography, in particular the Tibetan Plateau and the Andes, and similar detail is seen for EC-EARTH. As noted by Watterson et al. (2014), this detail may depend on the method used for extrapolating pressure to sea level beneath land, and EC-EARTH is evidently favored by the similarity of its atmospheric code to that of the reanalysis model for ERAInterim. In fact, over the GO domain, ACCESS1.0 (Bi et al. 2013) achieves a higher score. Its psl field (Fig. S3) appears rather smoother over Asia and South America, but that reduces the similarity to ERA-H.

\section{b. Variation between models}

Flato et al. (2013), in their Fig. 9.2c, presented the average over all available CMIP5 models of the absolute error in the climatological annual mean temperature relative to ERA-Interim. This was typically within $2 \mathrm{~K}$, but with larger values over high orography, some coasts, and regions with sea ice. Regardless of the observational dataset, differing skill scores will result from the variation in the climatological fields between the modelsseasonal fields in our case. Figure 2 shows the average across the four seasons of the intermodel standard deviation, with the SDs for the individual seasons shown in the supplementary figures. For tas, Fig. 2a, the SD is mostly 1.5 to $3 \mathrm{~K}$ over land, with higher values where there is steep topography, notably around Tibet, the Andes, and southern Greenland. Larger variation is seen over polar oceans, especially in DJF (Fig. S4a in the supplementary material), when northern land also varies more. This pattern matches the occurrence of sea ice and snow, which varies across models (Flato et al. 2013).

Variation across the model simulations of precipitation (Fig. 2b), measured in millimeters per day, tends to be larger in the tropics, and in the seasons when rainfall is heavy (Fig. S5 in the supplementary material). The standard deviation peaks at over $5 \mathrm{~mm} \mathrm{day}^{-1}$ near the steep topography of the Himalayas, Indonesia, and the Andes. The representation of orography and its impact on moisture fluxes in the lower troposphere in models evidently contributes to such differences in rainfall. In the midlatitudes, both over land and ocean, the SD is typically $0.5-1 \mathrm{~mm} \mathrm{day}^{-1}$, which may be a considerable fraction of the mean there. The SD in psl (Fig. 2c) exceeds $3 \mathrm{hPa}$ in high latitudes (and $4 \mathrm{hPa}$ in winter, in each hemisphere; Fig. S6 in the supplementary material), declining to below $1 \mathrm{hPa}$ near the equator. The variation is largest, in each season, over high orography, especially the Tibetan and Greenland plateaus. This appears consistent with differences in the method of extrapolation to sea level, as noted before. 

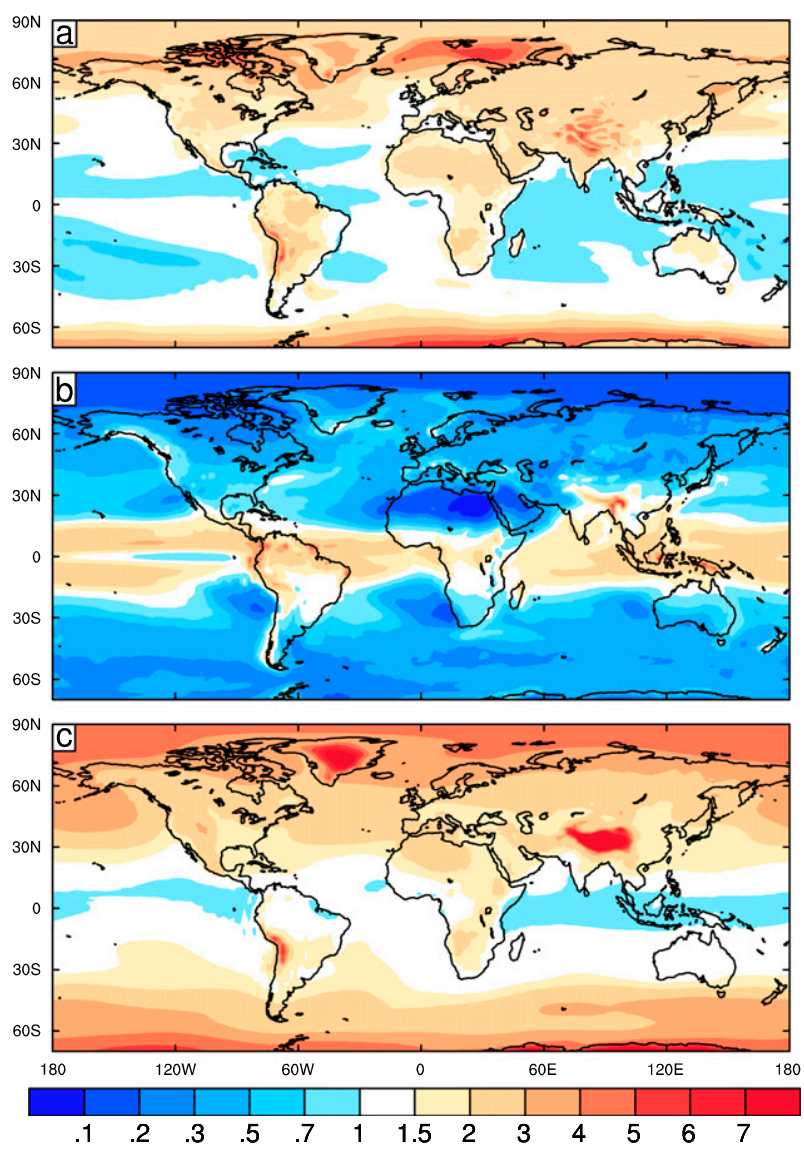

FIG. 2. Variation across the present (1975-2004) climate simulated by 42 CMIP5 models, after interpolation to the ERA-H $0.75^{\circ}$ grid. Shown is the standard deviation of the 42 means for each season, averaged over the four seasons, for (a) tas (K), (b) pr $\left(\mathrm{mm} \mathrm{day}{ }^{-1}\right)$, and (c) psl (hPa).

From Fig. 2, it is seen that the spatial pattern is rather different between tas, pr, and psl, although there are common peaks in regions of high orography. The skill tests used here, depending on absolute differences (and not percentage differences in rainfall, for instance), will be influenced by such regional variation. Some of the continental domains considered by Watterson et al. (2014) may avoid the main peaks, but they are potentially influenced by contrasts associated with the representation of land and ocean as suggested in the introduction. Across much of the global coastline, there are contrasts in the climatological fields. If a model coastline differs from that in the observational dataset, more likely in a coarse-resolution model, then this can generate a bias. In Fig. 2, there are often sharp contrasts between adjacent land and ocean SD values. Variation in tas is usually larger over land, except in polar regions, and not specifically near coasts. Variations in pr and psl tend to be larger over near-coastal land with high orography, such as Indonesia and the Andes. Along some coasts, such as the southwest of South America, the western North America, there is enhanced variation in pr over both ocean and land. This seems related to the mean intensity of pr there. In all, effects of differing model resolution on the intermodel SD do not seem particularly focused on coasts. This suggests that there will not be a major effect of resolving coasts on scoreresolution relationships, even for the smaller continental domains. In any case, this can be explored through comparisons with the larger ocean and global domains.

With respect to the overall improvement from CMIP3 to CMIP5, the variation among the 24 CMIP3 models is of interest. The CMIP3 SD fields (not shown) have a similar pattern to those of CMIP5. The ratio of CMIP5 to CMIP3 of the four-season average fields is shown for each variable in the supplementary material (Fig. S7). The average over GLO of the ratio for psl is 0.73 , with pressures in the tropics, especially, being more consistent in CMIP5. In fact, this average ratio is raised to 0.90 if one CMIP3 model with a large bias in the global mean psl is omitted. Temperatures are somewhat more consistent in CMIP5, with an average ratio of 0.92 . The average for pr is merely 0.97 . Indeed, the variation in $\mathrm{pr}$ among models is often larger in CMIP5, particularly over regions with high variation in Fig. $2 \mathrm{~b}$. This indicates that over such locations there may be more CMIP5 models with low agreement with a particular observational dataset, than for CMIP3, which would have implications for global skill scores.

\section{Skill assessments using the $M$ metric}

To quantify the overall skill level of a model, following Watterson et al. (2014), the $M$ values for similarity to the ERA-H climatological data have been averaged over the four seasons. The newer CMIP5 models feature strongly in the list, given in Table 2, of the top scoring model (or simply "top model") for each of the variables and domains, out of the combined CMIP3 and CMIP5 ensemble of 66 models. The four-season average $M$ scores for all models are shown as a function of model grid length for various cases in Figs. 3, 4, and 5. In these plots three categories of models are indicated: the CMIP5 models divided into the AO and ES categories, and the CMIP3 models (all considered to be AO). Mean values of score and length for each category, and for CMIP5 as a whole, are shown with enlarged points. Regression lines for the score as a function of length are plotted, with that for the combined ensemble shown in black. The slope and corresponding correlation between score and length are also given in Table 2. The $r$ values are also marked in the plots. The other lines and the implications of the regression results will be discussed in the next section. 
TABLE 2. Results from skill score analyses for 66 models (42 CMIP5 and 24 CMIP3) compared to ERA-H (ERA-Interim on a $0.75^{\circ}$ grid), for several variables (Var) and over three domains from $60^{\circ} \mathrm{S}$ to $90^{\circ} \mathrm{N}$ : land and ocean, separately and combined. From scores determined by the $M$ statistic are the top model, slope $b$ of the regression line with model grid length (points per $100 \mathrm{~km}$ ), the corresponding correlation $r$ (multiplied by 100, for convenience), the difference between the mean scores for CMIP5, relative to CMIP3 $(\Delta)$, and the separation of the individual regression lines $(\delta$, see text). Also given are the correlations for $M$ applied to the smoothed data ERA-S $\left(r_{s}\right)$, and correlations for the metric rootmean-square difference $\left(r_{\text {rms }}\right)$. Below are the case of tas over GL, with model data adjusted by the global annual bias, and the "continental" test, with scores averaged over six continents and GL, and two datasets.

\begin{tabular}{llcccccc}
\hline Var & \multicolumn{1}{c}{ Top model } & $b$ & $r$ & $r_{s}$ & $\Delta$ & $\delta$ & $r_{\text {rms }}$ \\
\hline Land (GL domain) & & & & & & \\
tas & CESM1(BGC) & -23 & -61 & -57 & 28 & 16 & 57 \\
pr & CCSM4 & -36 & -59 & -54 & 25 & 1 & 38 \\
psl EC-EARTH & -70 & -56 & -55 & 37 & -12 & 52 \\
Avg & CESM1(CAM5) & -43 & -67 & -64 & 30 & 2 & 66 \\
Ocean (GO domain) & & & & & & \\
tas & MPI-ESM-MR & -16 & -43 & -42 & 22 & 13 & 40 \\
pr CanESM2 & -24 & -35 & -34 & 29 & 15 & 19 \\
psl ACCESS1.0 & -50 & -54 & -54 & 51 & 21 & 52 \\
Avg CESM1(CAM5) & -30 & -55 & -55 & 34 & 17 & 55 \\
Land and ocean (GLO domain) & & & & & \\
tas CESM1(BGC) & -18 & -53 & -51 & 24 & 14 & 49 \\
pr CESM1(CAM5) & -27 & -45 & -42 & 27 & 11 & 27 \\
psl CESM1(CAM5) & -55 & -56 & -56 & 46 & 11 & 54 \\
Avg CESM1(CAM5) & -34 & -61 & -60 & 32 & 12 & 62 \\
Land (GL domain, bias adjusted) & & & & & \\
tas CESM1(CAM5) & -21 & -71 & - & 17 & 4 & - \\
Continental (two datasets) & & & & & & \\
Avg EC-EARTH & -51 & -73 & - & 37 & 4 & - \\
\hline
\end{tabular}

The model with the highest score in the case of the three-variable average score over land (GL; Fig. 3a) is CESM1(CAM5) (an AO model, length $96 \mathrm{~km}$; to aid identification on the plot), followed by EC-EARTH (AO, $100 \mathrm{~km})$. CESM1(CAM5)'s advantage, 6 points, is greater than the statistical uncertainty in such scores of typically 3 points, given the SD of the CSIRO Mk3.6 values, which is 1.5 points for GL. Not only do the two models have high resolution, but their points lie well above the regression line, as does the third ranked model CNRM-CM5 (AO, $125 \mathrm{~km}$ ) (Voldoire et al. 2013). The points for the two most highly resolved models, MIROC4h (AO, $50 \mathrm{~km})$ and CMCC-CM (AO, $67 \mathrm{~km}$ ), are closer to the line. The correlation for this case ("Avg" in Table 2), -0.67 , is the highest of all the ERA-H results, while those for the individual variables remain moderately high. All four top models for GL listed in Table 2 have high resolution (length $100 \mathrm{~km}$ or better). The pr case is considered further in section 6 .

As detailed in the supplementary material, Watterson et al. (2014) combined results for the GL domain with
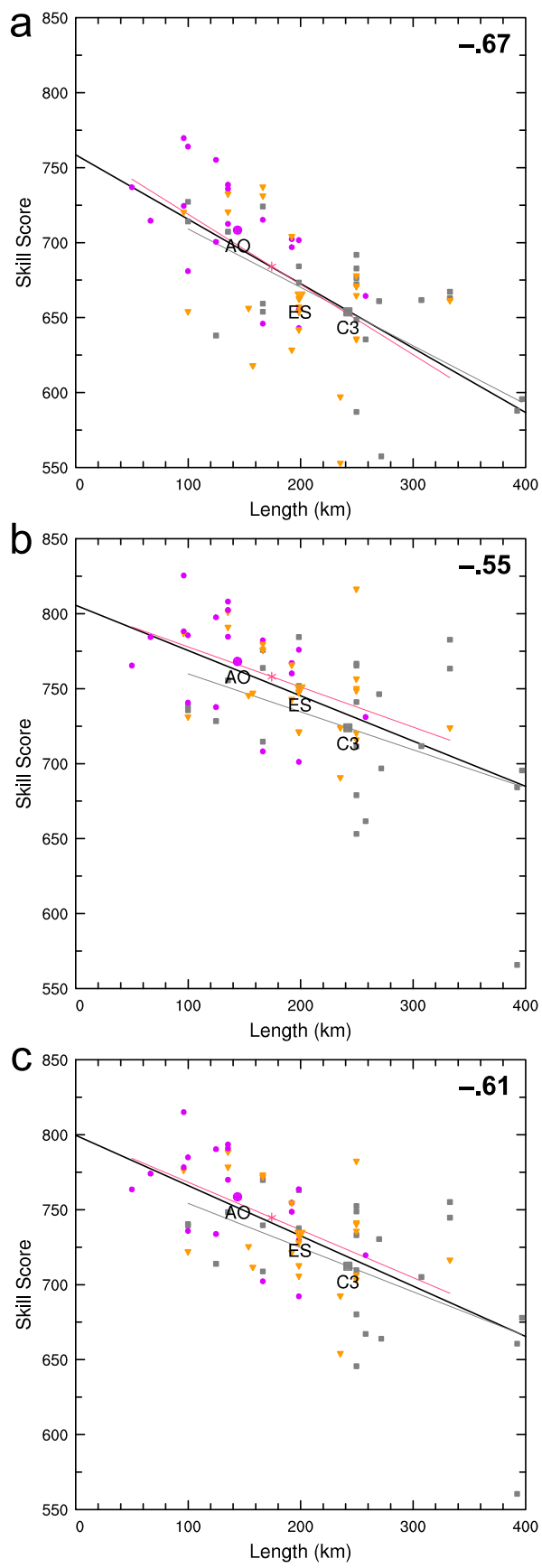

FIG. 3. Three-variable average $M$ skill score ( $y$ axis) and model resolution (using grid lengths; $x$ axis) from 66 models, and for three domains (as in Table 1): (a) land, (b) ocean, (c) combined land and ocean, all for the ERA-H data. The 24 CMIP3 (C3) models are represented by gray squares. The 42 CMIP5 models are partitioned into ES (orange triangles) and AO (purple circles) categories. The mean values are shown with larger symbols, as labeled. The CMIP5 mean is shown with a pink star. Regression lines for scores approximated as a linear function of length are shown for the combined ensemble (black), and the individual CMIP3 (gray) and CMIP5 (pink) ensembles. The correlation coefficient $r$ for the combined ensemble is marked in the top-right corner. One low score (522) is not shown in (a). 


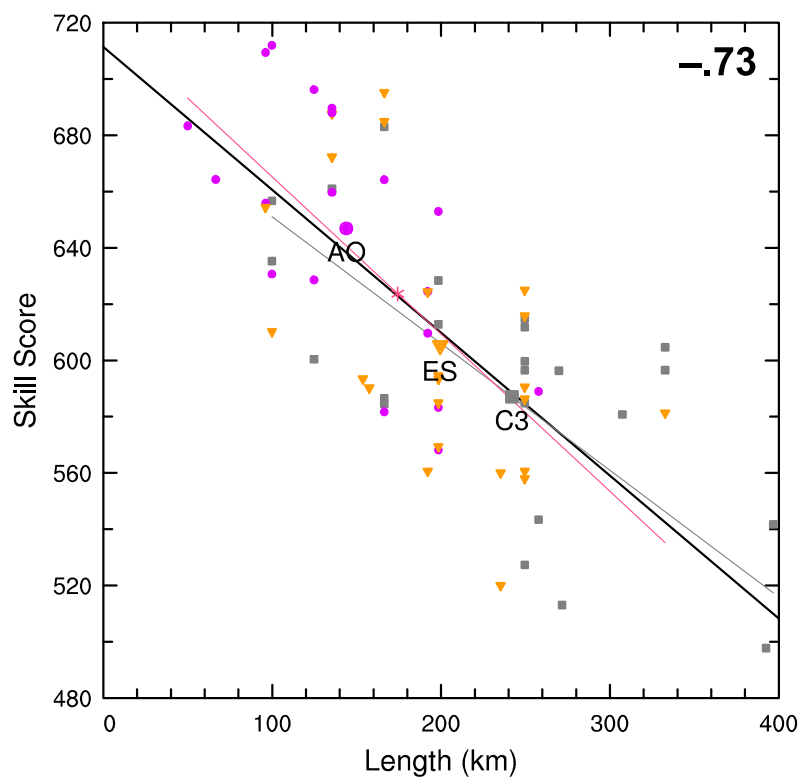

FIG. 4. As in Fig. 3, but for the overall $M$ skill scores for the continental test, using both ERA and CRU datasets.

those for six continents within it. The scores averaged over these seven domains and over the two datasets ERA and CRU (Table 1) are denoted here the "continental" test or case, with the results plotted in Fig. 4. In this test for land, EC-EARTH $(\mathrm{AO}, 100 \mathrm{~km})$ has the highest score (Table 2), just surpassing CESM1(CAM5), on account of better scores for CRU (see values for GL in Tables S1 and S2). The relative ranking for the two models is again confirmed by scores from the additional simulations. The average score for the 42 CMIP5 models is raised to 624 points, from the 618 points for the 25 -member average from Watterson et al. (2014), which compares with the CMIP3 average of 587. The regression line is barely changed, with the overall correlation strengthened slightly to -0.73 (from -0.71 ; section 1 ), and is higher than for the GL domain ERA-H result (Table 2). This holds also for the correlations for each variable (not given).

The scores with ERA-H for the ocean domain GO (Fig. 3b) tend to be higher than those for land on account of the larger spatial variation of the fields (and hence denominator in the formulation for $M$ ). Together with somewhat lower correlations with resolution, the regression slopes are lower than for GL. For the three variables, the leading models (Table 2) are different from those for land, and CanESM2 (Chylek et al. 2011) has a relatively large grid length of $250 \mathrm{~km}$. EC-EARTH $(\mathrm{AO}, 100 \mathrm{~km})$, in particular, does not score as well relative to other models over GO. Nevertheless, CESM1(CAM5) (AO, $96 \mathrm{~km}$ ) is again clearly top for Avg (Fig. 3b), with its lead being large compared to the estimated uncertainty of 5 points.
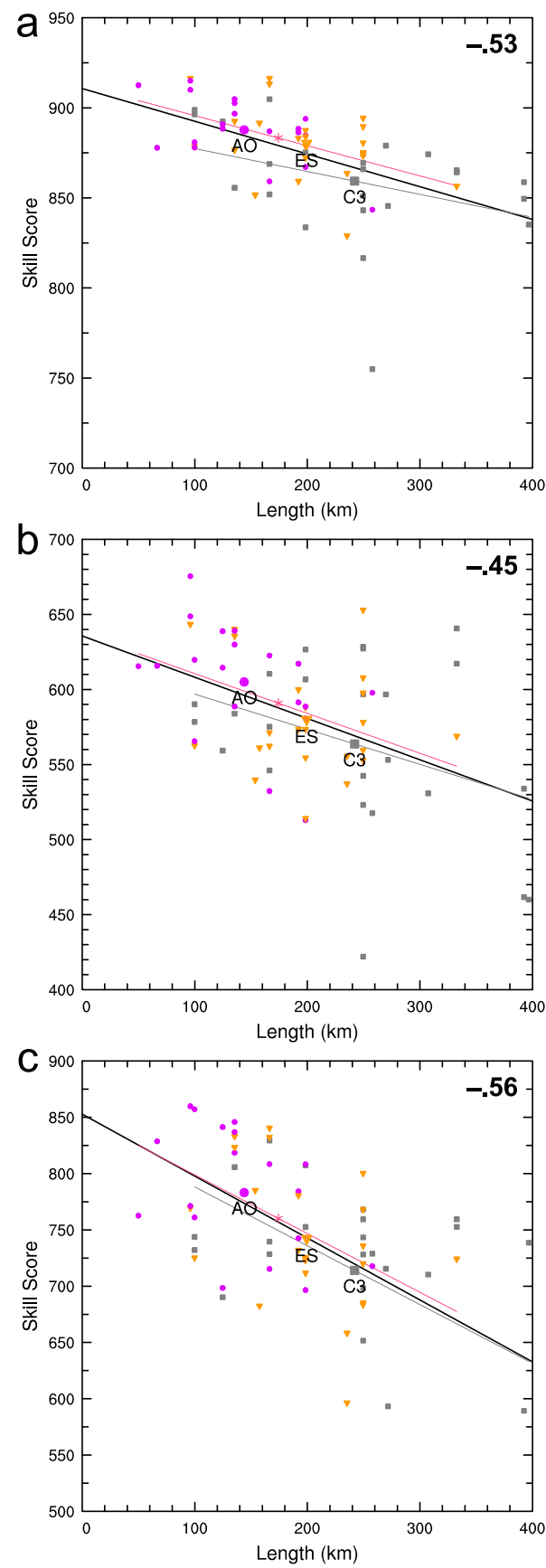

FIG. 5. As in Fig. 3, but for the GLO domain, and individual variables compared to ERA-H: (a) tas, (b) pr, and (c) psl. One score (370) is omitted from (c).

The results in Table 2 for the combined domain GLO are all intermediate between those for GL and GO. The plot for the three-variable average scores in Fig. 3c shows a substantial superiority for CESM1(CAM5) (22 points; see Tables S1 and S2 for values, with the estimated uncertainty being 4 points). The plots for the individual variables over this domain, Fig. 5 , show that this 
results from all-around performance, but particularly for $\mathrm{pr}$ (Fig. 5b). For tas (Fig. 5a) the ES model, CESM1(BGC), also at length $96 \mathrm{~km}$, is listed as top, from a single simulation, but allowing for uncertainty (estimated at 4 points) it is matched by four other models, with CESM1 (CAM5) following closely behind them. For psl (Fig. 5c), CESM1(CAM5) is matched by EC-EARTH. For each domain, the slope of the line (Table 2) is larger for psl than for tas, partly as a result of the larger range of $M$ values for psl (see Watterson et al. 2014). The higher average psl score for CMIP5, compared to CMIP3, is also consistent with the lower variation in psl across the CMIP5 simulations, as noted in the previous section.

\section{Influences on the scores and rankings}

\section{a. Model resolution}

Based on the correlations between $M$ skill scores and model resolution for the 66-model ensemble, resolution relates to typically $40 \%$ of the variance in the scores. Hence, resolution seems to have an influence on model skill, and this holds also on a somewhat broader scale, as evidenced by the consideration of observational data at lower resolution in the continental test.

For a collection of models with similar resolution, the difference in skill scores may indicate a further influence of model formulation, as noted by Watterson et al. (2014). For instance, in Fig. 3a, CESM1(CAM5) and EC-EARTH have scores well above others with grid length $100 \mathrm{~km}$ or less. Potentially, these models benefit from development of the modeling system that has improved their skill with respect to this particular test. Such development can include higher vertical resolution of the model atmosphere; see, for example, Karpechko et al. (2008). Some models simulate the ocean in greater detail than given by the data grid used here (see Flato et al. 2013). The inclusion of ES components do not appear to improve the agreement with ERA-H for tas, $\mathrm{pr}$, and psl, even for related models run at the same resolution. Indeed, CESM1(BGC) $(\mathrm{ES}, 96 \mathrm{~km})$ scores less well overall than CESM1(CAM5) (AO, 96 km).

In most cases, the plotted category mean points lie on or close to the regression line for the 66-model ensemble. Thus, as for the tests for the continents in the smaller ensemble, the improvement from CMIP3 to CMIP5 given for each case in Table 2 (represented by $\Delta$ ) seems largely consistent with the change expected from the regression slope coefficient ( $b$ in Table 2) combined with the difference in mean grid length of $67.6 \mathrm{~km}$. For instance, $96 \%$ of the increase for the GL Avg case is "explained" by the regression relationship, as
Watterson et al. (2014) put it. The remaining difference, given by the combined offsets of the two mean points relative to the regression line, is barely one skill point.

Formal assessment of the statistical significance of the regression results is hampered by the interdependence of the model codes (e.g., Knutti et al. 2013) and the nature of this combined "ensemble of opportunity." If the number of models considered independent is (crudely) estimated as half the total (given the numerous pairs of model versions), a correlation of -0.39 is significantly different from zero at the 0.02 level (e.g., Yamane 1967). The results discussed so far exceed that value, other than pr for GO. The $90 \%$ confidence interval for the Fig. 3c case, centered on $r=-0.61$, is estimated from -0.77 to -0.38 . Other statistics also contain statistical uncertainty and may differ for a different selection of models. In any case, Watterson et al. (2014) noted that correlations between scores and model length calculated from the available 25 CMIP5 models alone were usually similar to those for the combined ensemble. In fact, for the present tests, this tends to hold for both the CMIP3 and CMIP5 categories. Further, as seen in the plots, the individual regression lines are also mostly similar to the combined result. This is reassuring, with regard to the relationship with resolution. It would be possible for an ensemble that combined two sets of points that were quite distinct to produce a sloped regression line, even if there was no correlation within each set. The combined result could be merely a coincidence. It may be expected, nevertheless, that some improvement from CMIP3 to CMIP5 has followed from the development of modeling techniques, separate from that of horizontal resolution alone.

A statistical theory that considers whether a single regression line is an adequate fit to two sets of data points was presented by Brownlee (1960, section 11.6 therein). Even when the slopes of the individual regression lines are similar there may be a separation of the lines. This can be interpreted as a difference in the mean of the dependent variable (skill here) that is additional to one resulting from the difference in the mean of the independent variable (grid length). Our CMIP3 and CMIP5 regression lines can be used to give an indication of this effect. The skill values on the lines at the overall mean grid length $(199 \mathrm{~km}$, which happens to be close to the ES average) are suitable for this estimation. The separation of the lines there is included in Table 2 (as $\delta$ ). For both the overall continental scores (Fig. 4) and for the new GL Avg case (Fig. 3a) the individual regression lines almost overlie the combined result. For GL Avg the difference ( $M$ value) in the lines at the mean length is merely two skill points, or $7 \%$ of the 
improvement $(\Delta)$. As with the offsets of the mean points from the combined regression line, above, there is little direct indication of improvement other than through resolution.

For both the ocean (GO, Fig. 3b) and global (GLO, Fig. 3c) domains there is a larger separation between the individual regression lines. This represents $49 \%$ (GO) and $37 \%$ (GLO) of the improvement in CMIP5, relative to CMIP3. Turning to the global results for each variable (Fig. 5), the largest separation, both in points and percentage of the improvement, is for tas. Similar separations, of 13-16 points, occur for tas in the land and ocean cases in Table 2 and the continental case (not shown). These improvements are typically $20 \%-30 \%$ larger than those calculated using the offsets from the overall regression line because the slopes of the individual lines tend to be a little smaller. In these cases, the percentage of improvement explained by resolution from the overall line might therefore be considered an overestimate. Over land, the improvement in skill scores for pr and psl from CMIP3 to CMIP5 is also substantial. For $\mathrm{pr}$, the individual lines almost coincide, and the separation is merely one point. For psl, $\delta$ is actually negative for this set of models, although relatively small compared to $\Delta$. The three variables combine to produce the consistency of the average scores with the 66-model regression line (Fig. 3a).

The scores for tas appear to provide a clear indication of improvements that are in addition to those from resolution. Further analysis suggests that these are partly due to improved agreement of the global mean temperature to that in ERA-Interim. Results are included in Table 2 for the assessment of the seasonal climatologies of tas over GL after each model field has been "bias adjusted" by a constant, equal to the difference in the global annual mean of ERA-Interim and the model. The CMIP3 mean score improves by 18 points, compared to only 5 for CMIP5. This relates to the larger bias in the global mean, which averages $1.1 \mathrm{~K}$ for CMIP3, compared to $0.6 \mathrm{~K}$ for CMIP5. The separation between the individual regression lines is reduced to 4 points, compared to 16 for the nonadjusted data. The overall correlation coefficient increases, consistent with a stronger relationship with resolution.

\section{b. Dependence on metric}

The tests against ERA-H have been repeated using rmse as an alternative metric to $M$. Plots of values for the GLO domain and each variable are shown in Fig. 6. The rankings of models based on rmse are mostly similar to those for $M$. Aside from the different units, typical values of rmse are similar for the three variables,
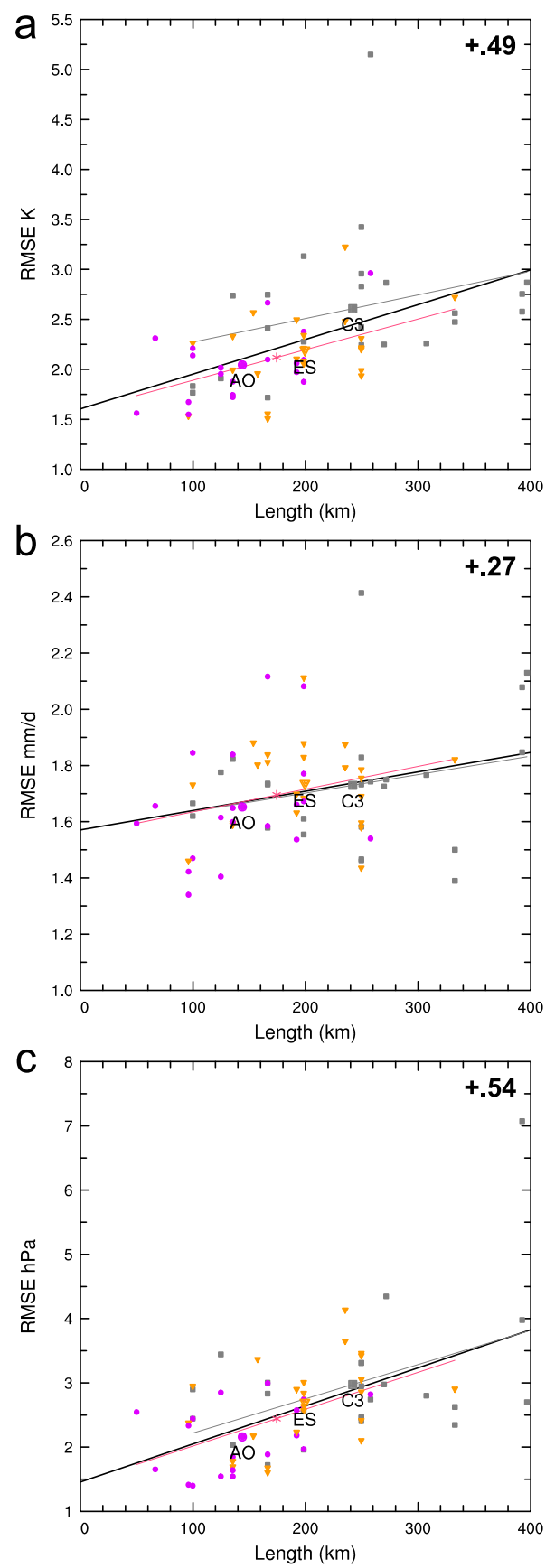

FIG. 6. Average root-mean-square error ( $y$ axis) and model resolution (using grid lengths; $x$ axis) from 66 models compared to ERA-H, for the GLO domain: (a) tas, (b) pr, and (c) psl. Symbols are as in Fig. 3. Also shown are regression lines for rmse approximated as a linear function of length.

which encourages averaging of these values also. CESM1(CAM5) is the top model from this averaging for GLO, as well as for GL and GO (matching its success for $M$ ). The correlations with length, given in Table 2 (as $\left.r_{\text {rms }}\right)$, are also nearly as large in magnitude as those for $M$. 
These results are to be expected given the link between $M$ and rmse (section 2c). However, there is more dependence on the metric in the case of pr, with the correlation with length falling to 0.19 for GO. Further tests indicate that the presence of the model variance $V_{X}$ in the formulation of $M$ leads to much of the difference. If the model variance and mean are replaced by those of the observational field (except in a few cases where this prevents the arcsine transformation), then these modified $M$ scores are almost linearly related to rmse, and the correlation with length closely matches that for rmse. The regression lines for rmse with length for CMIP3 and CMIP5, calculated separately, are mostly similar (Fig. 6). As for $M$ (Fig. 5a) the separation of lines for the tas case is clear. The lower correlation for pr corresponds to only a small improvement of CMIP5 from CMIP3 (Fig. 6b), when compared to ERA-H, with the average rmse for the ES models higher, despite a lower average grid length. The use of mae as the metric produces similar correlations (not given) with resolution to those in Table 2 for the Avg and psl cases. The correlations for tas are a little lower than for $M$ and rmse, and intermediate between them for pr.

\section{c. Observational data}

While the use of the ERA-H data, on a finer grid, rather than ERA has little effect on skill scores, spatial smoothing of data or aggregation of values can change a score because of the lower effective resolution. A further set of correlations is given in Table 2, following from the use of smoothed ERA-H fields, denoted ERA-S. The smoothing consists of replacing each grid point value with the average of it and the adjacent four values, applied twice. This reduces the potential advantage of a high-resolution model in matching detail in the observational field. The $M$ scores are usually a little higher for the smoothed fields, but less so for some higher-resolution models. As a result the correlation tends to be lower. The decrease is largest for pr $(0.05)$ and tas (0.04) over GL.

The two sets of scores using the CRU and ERA datasets over land provide a further indication of sensitivity. Focusing on the GL results, the correlation across all 66 models between CRU and ERA for the three-variable average is 0.95 . However this falls to 0.86 for pr, and the correlation with model grid length is lower, at -0.38 , for CRU. If the CRU-H data are used, the correlation for pr over GL improves to -0.42 . However, there are differences in the points designated as land (see Fig. S1) between ERA and CRU, so the dependence of the pr data alone should be considered further.

\section{Further assessment of precipitation fields}

\section{a. Regional example}

The precipitation example shown in Fig. 1, for September-November, provided a specific example of dependence on the dataset, with differing scores for CESM1(CAM5) against ERA-H and GPCP-H. A regional example based on this case is instructive. The region bounded by $15^{\circ} \mathrm{S}-10^{\circ} \mathrm{N}$ and $82^{\circ}-73^{\circ} \mathrm{W}$ encompasses both high and low extremes in pr for SON, but with rather contrasting fields from the two models and four datasets shown in Fig. 7. Also shown are the multimodel mean (MMM) from each of CMIP5 and CMIP3, calculated from data interpolated to the ERA-H grid. The fields for CESM1(CAM5), ERA-H, and CRU-H have similarity in the sharp peak of pr along the coast of Colombia. Lower peaks are evident in both GPCP and GPCP-H, the CMCCCMS model (AO, $166 \mathrm{~km}$ ) and the two MMM fields. There are contrasts also in the very dry part of the domain.

Both $M$ and rmse scores for the region (land and ocean) for various model-observation pairs are given in Table 3. For both metrics, CESM1(CAM5) has the best score for all models against ERA-H, while CMCC-CMS has the best scores for this domain against both versions of GPCP. As before, the scores improve when ERA-H is replaced by the smoothed version ERA-S (Table 3), with that for CESM1(CAM5) improving by the least amount. Both models produce better scores than the MMM fields, with the exception of CESM1(CAM5), with GPCP using the rmse. In contrast, for the global domains, the CMIP5 MMM was found to be better than any individual model by Flato et al. (2013, their Fig. 9.7) and Watterson et al. (2014). The MMM for CMIP5 has better scores than that for CMIP3, with the exception of the rmse result for GPCP and GPCP-H. Clearly a high-resolution model can improve on the MMM, if the observational data show similar detail.

In general, the relative rankings of these four model fields are consistent with the detail in pr over this region, with CESM1(CAM5) favored by comparison with ERA-H, while the smoother model fields are favored by comparison with GPCP, at both resolutions. While comparison of observational datasets is beyond the scope of this analysis, the GPCP fields, even in the GPCP-H version, have smaller peaks and spatial standard deviation than the ERA-H fields. The comparison with CRU in this region suggests that this is not necessarily realistic, and Yatagai et al. (2012) provide a similar contrast for the Himalayan region.

\section{b. Global land analysis}

Contrasts between scores for the different models, datasets, and metrics similar to those for the region can 

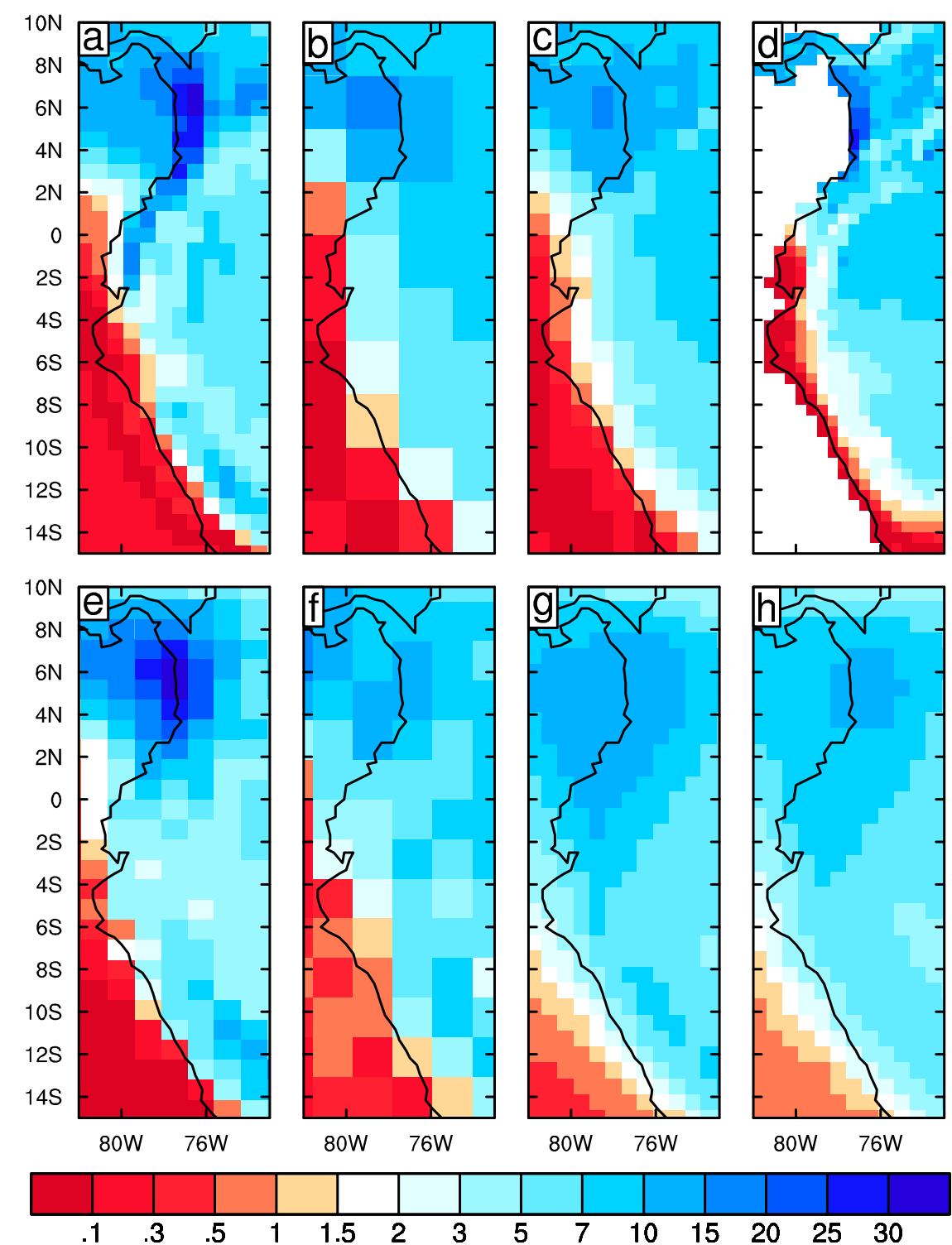

FIG. 7. Climatological mean pr for SON $\left(\mathrm{mm} \mathrm{day}^{-1}\right)$, as Fig. 1, but with detail from the far northwest of South America and for observational data from (a) ERA-H, (b) GPCP, (c) GPCP-H, and (d) CRU-H; model data from (e) CESM1(CAM5) and (f) CMCC-CMS; and the multimodel mean of the fields from (g) CMIP5 and (h) CMIP3.

be seen in the results already shown for the global domains and four-season averages. With climate impacts in mind, we can focus on results for pr over the land. The four-season average scores for all models are shown in Fig. 8, for two metrics and both ERA-H and GPCP-H data. The analysis for GPCP-H is done using the points on its $1^{\circ}$ grid that are within the land squares of ERA-H. Statistics for the ERA-H cases are already included in Table 2 and provide slightly stronger links to length than the GLO results shown in Figs. $5 b$ and $6 b$. The correlations (marked on Fig. 8) and regression line slopes are weaker in the GPCP-H case, -0.41 for $M$ and +0.19 for rmse. In fact, they are somewhat weaker again for GPCP over GLO and GO (not shown, but details for all GPCP-H cases are included in Table S3 of the supplementary material). For rmse over GL, there are particularly large values for two CMIP5 models (GISS-E2-H-CC, ES $198 \mathrm{~km}$, and GISS-E2-H, AO $198 \mathrm{~km}$ ). These models have some very high peaks at points in the tropics near steep topography (see Fig. 2b). The corresponding $M$ values are low, but moderated by the spatial SD terms, especially for ERA-H (Fig. 8a).

While the CMIP5 average rmse for ERA-H over GL remains slightly lower than for CMIP3, it is actually $1 \%$ 
TABLE 3. Skill scores for the case of pr in SON for the regional domain (land and ocean) and data shown in Fig. 7. Results are given for two metrics, $M$ and rmse, and for four observational fields. The two models used have the highest scores against these (by either metric). The scores for the MMM of the model fields from both CMIP5 and CMIP3 are given.

\begin{tabular}{lcccc}
\hline \hline & \multicolumn{4}{c}{ Observational data } \\
\cline { 2 - 5 } Model & ERA-H & ERA-S & GPCP & GPCP-H \\
\hline$M$ (points) & & & & \\
CESM1(CAM5) & 671 & 726 & 589 & 545 \\
CMCC-CMS & 483 & 562 & 749 & 745 \\
CMIP5 MMM & 455 & 518 & 516 & 503 \\
CMIP3 MMM & 379 & 439 & 492 & 485 \\
rmse (mm day ${ }^{-1}$ ) & & & & \\
CESM1(CAM5) & 3.18 & 2.55 & 3.43 & 3.91 \\
CMCC-CMS & 3.92 & 3.14 & 1.56 & 1.56 \\
CMIP5 MMM & 4.09 & 3.41 & 2.97 & 3.04 \\
CMIP3 MMM & 4.34 & 3.67 & 2.77 & 2.80 \\
\hline
\end{tabular}

higher for GPCP-H. The effect of the weak positive correlations with length in Fig. 8d (for each regression line) is more than overcome by the separation of the individual ensemble lines, with that for CMIP5 shifted toward larger rmse. The slightly reduced rmse skill for this CMIP5 ensemble, compared to CMIP3, holds for the GLO domain also (Table S3). While these details will depend on the actual selection of models, it is apparent that the combination of the GPCP-H data and the rmse metric tends to not favor some higher-resolution CMIP5 models. In all these cases, results for the lower-resolution GPCP are rather similar to those for GPCP-H. For example, the correlation with length for GL is -0.37 for $M$ and 0.14 for rmse, both only slightly weaker than in Figs. 8b and $8 \mathrm{~d}$. In addition to spatial detail, larger-scale systematic differences between GPCP (on both grids) and ERA-H, as evident in Fig. 1, are also important to the contrasts in scores.

As noted in the introduction, this dependence of precipitation skills on the metric and observational dataset is a major issue in weather forecasting. The improvement in apparent realism with higher resolution does not necessarily hold when an objective metric like rmse is used. Wernli et al. (2009) and others (see references therein) have developed alternative metrics, often involving error relative to the spatial detail in the model field. While position errors in climatological precipitation features may relate to topography and ocean surface temperatures, rather than timing of weather systems, the issues are similar. Further, if the observational field is relatively smooth, a high-resolution model can be penalized for its detail.

While further assessment of these issues is warranted, this analysis provides some understanding of the range of correlations with rainfall. It is shown that several of the high-resolution CMIP5 models simulate much of the regional detail in climatological precipitation that occurs in ERA-Interim. This detail may not be wholly realistic, and the similar correlation of the $M$ score with length to that for tas and psl may not be truly reflective of skill in simulating pr at higher resolution. However, the contrary result that the 42-model CMIP5 ensemble simulates GPCP pr with less accuracy than CMIP3 seems unduly pessimistic.

\section{Conclusions}

The inclusion of 17 newer CMIP5 models to the ensemble analyzed by Watterson et al. (2014) increases the superiority of CMIP5 over CMIP3 with respect to the simulation of present climate over global land (excluding Antarctica). As quantified by the ArcsinMielke skill score $(M)$, two of the new models with higher resolution, EC-EARTH and CESM1(CAM5), have the highest two scores (averaged over tas, pr, and psl, and over four seasons) for that domain. These models provide examples of simulated seasonal climatologies that reproduce well not only the global observational fields, but also some regional detail at the grid point scale. The correlation between the combined scores (for six continental domains and two datasets) and model grid length in the new 66-model ensemble is -0.73 .

The new analyses here show that the skill of CMIP5 extends to the ocean and global domains. Correlations between skill scores and model horizontal resolution are nearly as strong over the ocean, indicating that the relationship for land is not simply due to the better resolving of coastlines and orography. For the global domain (excluding the Antarctic cap) CESM1(CAM5) has the best overall match to the ERA-Interim climatology. The correlation over the ensemble of the average $M$ score with grid length is -0.61 , and it is around -0.5 for each of the three variables. The correlations are lower in magnitude if the root-meansquare difference (rmse) is used as the metric, particularly for pr. This follows from the scaling of the error by spatial variation in $M$ (and also $r$, used as a metric for fields). The relationship with length is also weaker if the relatively smooth GPCP observational dataset is used.

The differing scores for models follow from the variations in the simulated fields across the ensemble. These variations tend to be larger over land than ocean, but not specifically near coasts. Variations in pressure are largest over some regions of high orography, while 

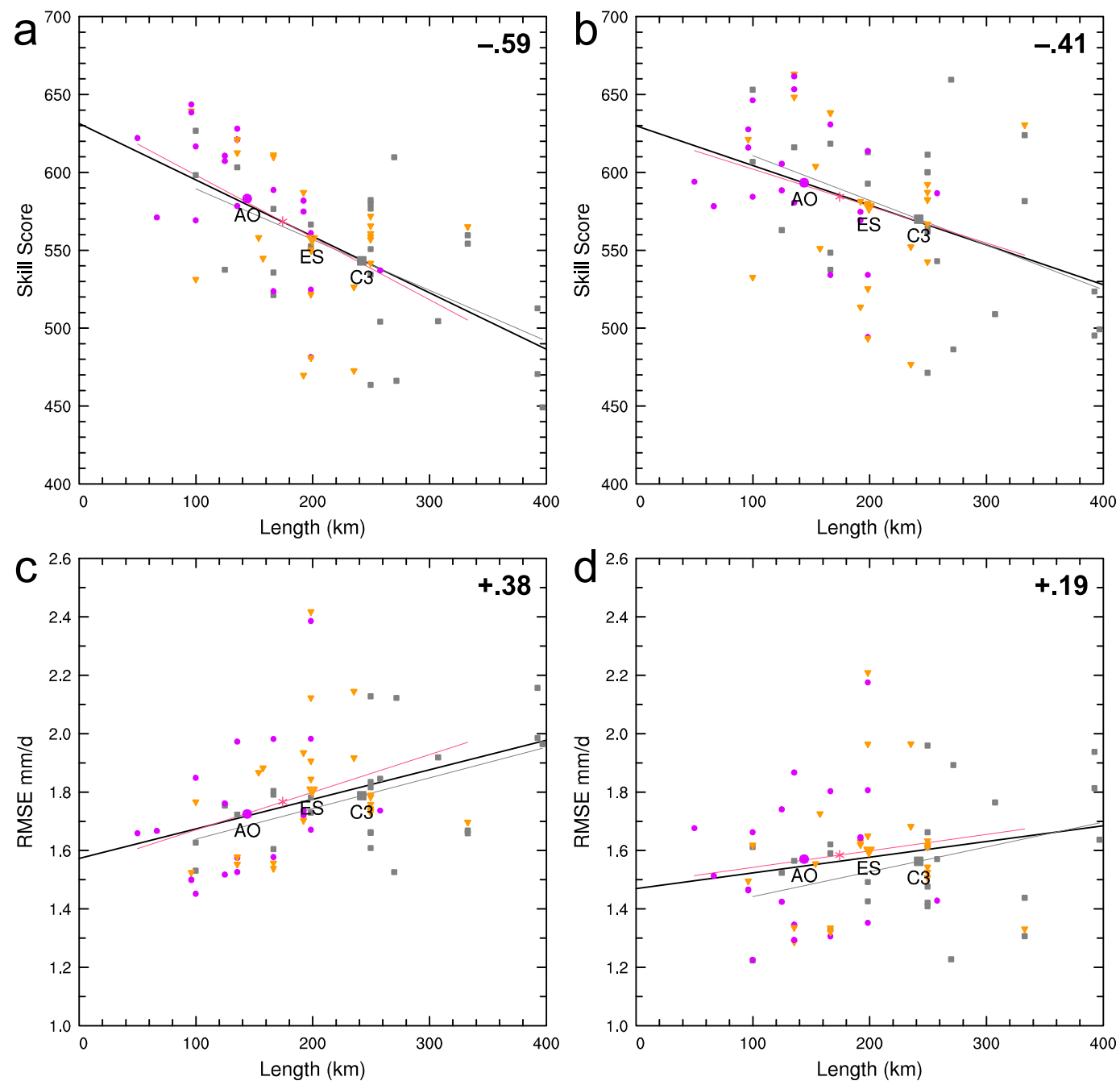

FIG. 8. As in Fig. 3, for the land (GL domain) and pr using both skill measures and two observational datasets: ERA-H (a) $M$ and (c) rmse and GPCP-H (b) $M$ and (d) rmse.

those in temperature are often large in regions of strong topographic variation, including the Arctic. Variation in the simulation of rainfall is largest in the wet tropics. The ability of some higher-resolution models in simulating regional detail in temperature and rainfall over land, in particular, improves their scores, provided that such detail is present in the observational data.

In the plots of scores and grid length, the averages for the CMIP3 models, and for the partition of CMIP5 models into standard atmosphere-ocean models and those with enhanced Earth system components, all lie near the regression line for scores as a function of grid length over the combined ensemble. Thus for most tests the improved average score of CMIP5 models relative to that for CMIP3 is largely consistent with their increased resolution. However, the top scoring models tend to lie well above the regression line, pointing to additional gains that result from improved representation of physical processes, in these $\mathrm{AO}$ models, that are important to the seasonal averages tested. Clearly improvements in the simulation of climate can be sought through further development of model code as well as increased resolution. For precipitation, in particular, consideration of the observational data and metrics is an important component to the evaluation of skill.

Acknowledgments. This work was supported by the Australian Government's Regional Natural Resources Management Planning for Climate Change Fund. The author is grateful to the modeling groups, PCMDI, and others who established the CMIP5 archive, and to CAWCR colleagues who provided the $30-\mathrm{yr}$ model 
averages used, in particular Janice Bathols and Lawson Hanson. I thank three anonymous reviewers for their very helpful comments.

\section{REFERENCES}

Adler, R. F., and Coauthors, 2003: The version-2 Global Precipitation Climatology Project (GPCP) monthly precipitation analysis (1979-present). J. Hydrometeor., 4, 1147-1167, doi:10.1175/1525-7541(2003)004<1147:TVGPCP > 2.0.CO;2.

Bi, D., and Coauthors, 2013: The ACCESS coupled model: Description, control climate and validation. Aust. Meteor. Ocean J., 63, 41-64.

Boyle, J., and S. A. Klein, 2010: Impact of horizontal resolution on climate model forecasts of tropical precipitation and diabatic heating for the TWP-ICE period. J. Geophys. Res., 115, D23113, doi:10.1029/2010JD014262.

Brownlee, K. A., 1960: Statistical Theory and Methodology in Science and Engineering. Wiley, 570 pp.

Chylek, P., J. Li, M. K. Dubey, M. Wang, and G. Lesins, 2011: Observed and model simulated 20th century Arctic temperature variability: Canadian Earth System Model CanESM2. Atmos. Chem. Phys. Discuss., 11, 22 893-22 907, doi:10.5194/ acpd-11-22893-2011.

CSIRO and Bureau of Meteorology, 2015: Climate change in Australia Information for Australia's Natural Resource Management Regions. Technical report, CSIRO and Bureau of Meteorology, Australia, 216 pp. [Available online at http:// www.climatechangeinaustralia.gov.au/en/publications-library/ technical-report/.]

Dee, D. P., and Coauthors, 2011: The ERA-Interim reanalysis: Configuration and performance of the data assimilation system. Quart. J. Roy. Meteor. Soc., 137, 553-597, doi:10.1002/ qj.828.

Delworth, T. L., and Coauthors, 2012: Simulated climate and climate change in the GFDL CM2.5 high-resolution coupled climate model. J. Climate, 25, 2755-2781, doi:10.1175/ JCLI-D-11-00316.1.

Flato, G., and Coauthors, 2013: Evaluation of climate models. Climate Change 2013: The Physical Science Basis, T. F. Stocker et al., Eds., Cambridge University Press, 741-866.

Giorgetta, M. A., and Coauthors, 2013: Climate and carbon cycle change from 1850 to 2100 in MPI-ESM simulations for the Coupled Model Intercomparison Project phase 5. J. $A d v$. Model. Earth. Syst., 5, 572-597, doi:10.1002/jame.20038.

Gleckler, P. J., K. E. Taylor, and C. Doutriaux, 2008: Performance metrics for climate models. J. Geophys. Res., 113, D06104, doi:10.1029/2007JD008972.

Gruber, A., and V. Levizzani, 2008: Assessment of global precipitation products. WCRP-128, WMO/TD-No. 1430, 50 pp.

Hazeleger, W., and Coauthors, 2012: EC-EARTH V2.2: Description and validation of a new seamless Earth system prediction model. Climate Dyn., 39, 2611-2629, doi:10.1007/ s00382-011-1228-5.

Huffman, G. J., R. F. Adler, M. Morrissey, D. T. Bolvin, S. Curtis, R. Joyce, B. McGavock, and J. Susskind, 2001: Global precipitation at one-degree daily resolution from multisatellite observations. J. Hydrometeor., 2, 36-50, doi:10.1175/ 1525-7541(2001)002<0036:GPAODD > 2.0.CO;2.

Hurrell, J., and Coauthors, 2013: The Community Earth System Model. Bull. Amer. Meteor. Soc., 94, 1339-1360, doi:10.1175/ BAMS-D-12-00121.1.
Karpechko, A. Y., N. P. Gillett, G. J. Marshall, and A. A. Scaife, 2008: Stratospheric influence on circulation changes in the Southern Hemisphere troposphere in coupled climate models. Geophys. Res. Lett., 35, L20806, doi:10.1029/2008GL035354.

Knutti, R., D. Masson, and A. Gettelman, 2013: Climate model genealogy: Generation CMIP5 and how we got there. Geophys. Res. Lett., 40, 1194-1199, doi:10.1002/grl.50256.

Mass, C. F., D. Owens, K. Westrick, and B. A. Colle, 2002: Does increasing horizontal resolution produce more skillful forecasts? Bull. Amer. Meteor. Soc., 83, 407-430, doi:10.1175/ 1520-0477(2002)083<0407:DIHRPM > 2.3.CO;2.

Melillo, J. M., T. C. Richmond, and G. W. Yohe, Eds., 2014: Climate Change Impacts in the United States: The Third National Climate Assessment. U.S. Global Change Research Program, $841 \mathrm{pp}$.

Mielke, P. W., Jr., 1991: The application of multivariate permutation methods based on distance functions in the Earth sciences. Earth-Sci. Rev., 31, 55-71, doi:10.1016/0012-8252(91)90042-E.

Mishra, V., D. Kumar, A. R. Ganguly, J. Sanjay, M. Mujumdar, R. Krishnan, and R. D. Shah, 2014: Reliability of regional and global climate models to simulate precipitation extremes over India. J. Geophys. Res. Atmos., 119, 9301-9323, doi:10.1002/ 2014JD021636.

Neale, R. B., J. Richter, S. Park, P. H. Lauritzen, S. J. Vavrus, P. J. Rasch, and M. Zhang, 2013: The mean climate of the Community Atmosphere Model (CAM4) in forced SST and fully coupled experiments. J. Climate, 26, 5150-5168, doi:10.1175/ JCLI-D-12-00236.1.

New, M., M. Hulme, and P. Jones, 1999: Representing twentiethcentury space-time climate variability. Part I: Development of a 1961-1990 mean monthly terrestrial climatology. J. Climate, 12, 829-856, doi:10.1175/1520-0442(1999)012<0829: RTCSTC $>2.0 . \mathrm{CO} ; 2$.

Pope, V. D., and R. A. Stratton, 2002: The processes governing horizontal resolution sensitivity in a climate model. Climate Dyn., 19, 211-236, doi:10.1007/s00382-001-0222-8.

Rotstayn, L. D., S. J. Jeffrey, M. A. Collier, S. M. Dravitzki, A. C. Hirst, J. I. Syktus, and K. K. Wong, 2012: Aerosol- and greenhouse gas-induced changes in summer rainfall and circulation in the Australasian region: A study using singleforcing climate simulations. Atmos. Chem. Phys., 12, 63776404, doi:10.5194/acp-12-6377-2012.

Sakamoto, T. T., and Coauthors, 2012: MIROC4h-A new highresolution atmosphere-ocean coupled general circulation model. J. Meteor. Soc. Japan, 90, 325-350, doi:10.2151/jmsj.2012-301.

Scoccimarro, E., and Coauthors, 2011: Effects of tropical cyclones on ocean heat transport in a high-resolution coupled climate general circulation model. J. Climate, 24, 4368-4384, doi:10.1175/2011JCLI4104.1.

Sheffield, J., and Coauthors, 2013: North American climate in CMIP5 experiments. Part I: Evaluation of simulations of continental and regional climatology. J. Climate, 26, 92099245, doi:10.1175/JCLI-D-12-00592.1.

Simmons, A. J., K. M. Willett, P. D. Jones, P. W. Thorne, and D. Dee, 2010: Low-frequency variations in surface atmospheric humidity, temperature and precipitation: Inferences from reanalyses and monthly gridded observational datasets. J. Geophys. Res., 115, D01110, doi:10.1029/2009JD012442.

Uppala, S. M., and Coauthors, 2005: The ERA-40 Re-Analysis. Quart. J. Roy. Meteor. Soc., 131, 2961-3012, doi:10.1256/qj.04.176.

Voldoire, A., and Coauthors, 2013: The CNRM-CM5.1 global climate model: Description and basic evaluation. Climate Dyn., 40, 2091-2121, doi:10.1007/s00382-011-1259-y. 
Watterson, I. G., 1996: Non-dimensional measures of climate model performance. Int. J. Climatol., 16,379-391, doi:10.1002/ (SICI)1097-0088(199604)16:4<379::AID-JOC18>3.0.CO; 2-U.

J. Bathols, and C. Heady, 2014: What influences the skill of climate models over the continents? Bull. Amer. Meteor. Soc., 95, 689-700, doi:10.1175/BAMS-D-12-00136.1.

Wernli, H., C. Hofmann, and M. Zimmer, 2009: Spatial forecast verification methods intercomparison project: Application of the SAL technique. Wea. Forecasting, 24, 1472-1484, doi:10.1175/2009WAF2222271.1.
Yamane, T., 1967: Statistics: An Introductory Analysis. Harper International, $919 \mathrm{pp}$.

Yatagai, A., K. Kamiguchi, O. Arakawa, A. Hamada, N. Yasutomi, and A. Kitoh, 2012: APHRODITE: Constructing a long-term daily gridded precipitation dataset for Asia based on a dense network of rain gauges. Bull. Amer. Meteor. Soc., 93, 14011415, doi:10.1175/BAMS-D-11-00122.1.

Yin, L., R. Fu, E. Shevliakova, and R. E. Dickinson, 2013: How well can CMIP5 simulate precipitation and its controlling processes over tropical South America? Climate Dyn., 41, 3127-3143, doi:10.1007/s00382-012-1582-y. 